\title{
Genome-wide association study identifies new loci associated with risk of HBV infection and disease progression
}

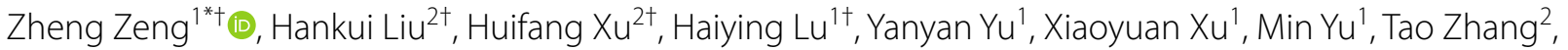
Xiulan Tian ${ }^{1}$, Hongli Xi ${ }^{1}$, Liping Guan², Jianguo Zhang ${ }^{2^{*}}$, Stephen J. O'Brien ${ }^{3,4^{*}}$ and the HBVstudy consortium

\begin{abstract}
Background: Recent studies have identified susceptibility genes of HBV clearance, chronic hepatitis B, liver cirrhosis, hepatocellular carcinoma, and showed the host genetic factors play an important role in these HBV-related outcomes.

Methods: Collected samples from different outcomes of HBV infection and performed genotyping by Affymetrix 500 k SNP Array. GCTA tool, PLINK, and Bonferroni method were applied for analysis of genotyping and disease progression. ANOVA was used to evaluate the significance of the association between biomarkers and genotypes in healthy controls. PoMo, $\mathrm{F}_{\mathrm{ST}}$, Vcftools and Rehh package were used for building the racial tree and population analysis. $\mathrm{F}_{\text {ST }}$ statistics accesses 0.15 was used as a threshold to detect the signature of selection.

Results: There are 1031 participants passed quality control from 1104 participants, including 275 HBV clearance, 92 asymptomatic persistence infection (ASPI), 93 chronic hepatitis B (CHB), 188 HBV-related decompensated cirrhosis (DC), 214 HBV-related hepatocellular carcinoma (HCC) and 169 healthy controls (HC). In the case-control study, one novel locus significantly associated with CHB (SNP: rs1264473, Gene: GRHL2, P $=1.57 \times 10^{-6}$ ) and HCC (SNP: rs2833856, Gene: EVA1C, $P=1.62 \times 10^{-6}$; SNP: rs4661093, Gene: ETV3, $P=2.26 \times 10^{-6}$ ). In the trend study across progressive stages post HBV infection, one novel locus (SNP: rs1537862, Gene: $L A C E 1, P=1.85 \times 10^{-6}$ ), and three MHC Ioci (HLA-DRB1, HLA-DPB1, HLA-DPA2) showed significant increased progressive risk from ASPI to CHB. Underlying the evolutionary study of HBV-related genes in public database, the derived allele of two HBV clearance related loci, rs3077 and rs9277542, are under strong selection in European population.

Conclusions: In this study, we identified several novel candidate genes associated with individual HBV infectious outcomes, progressive stages, and liver enzymes. Two SNPs that show selective significance (HLA-DPA1, HLA-DPB1) in non-East Asian (European, American, South Asian) versus East Asian, indicating that host genetic factors contribute to
\end{abstract}

\footnotetext{
*Correspondence: zeng@bjmu.edu.cn; zhangjg@genomics.cn;

lgdchief@gmail.com

†'Zheng Zeng, Hankui Liu, Huifang Xu and Haiying Lu contributed equally

to the manuscript

${ }^{1}$ Department of Infectious Diseases, Peking University First Hospital,

Beijing 100034, China

2 BGI-Shenzhen, Shenzhen 518083, China

${ }^{4}$ Guy Harvey Oceanographic Center, Halmos College of Natural Sciences

and Oceanography, Nova Southeastern University, Ft Lauderdale, FL

33004, USA

Full list of author information is available at the end of the article
} original author(s) and the source, provide a link to the Creative Commons licence, and indicate if changes were made. The images or other third party material in this article are included in the article's Creative Commons licence, unless indicated otherwise in a credit line to the material. If material is not included in the article's Creative Commons licence and your intended use is not permitted by statutory regulation or exceeds the permitted use, you will need to obtain permission directly from the copyright holder. To view a copy of this licence, visit http://creativecommons.org/licenses/by/4.0/. The Creative Commons Public Domain Dedication waiver (http://creativeco mmons.org/publicdomain/zero/1.0/) applies to the data made available in this article, unless otherwise stated in a credit line to the data. 
the ethnic disparities of susceptibility of HBV infection. Taken together, these findings provided a new insight into the role of host genetic factors in HBV related outcomes and progression.

Keywords: HBV infection, Disease progression, GWAS, Host genetic factors, SNPS

\section{Background}

Hepatitis B virus (HBV) infection is one of the most common infectious diseases, with about 248 million HBsAg positive individuals worldwide and the largest HBsAg positive population in China [1]. HBV infection can develop a wide spectrum of liver diseases, including chronic hepatitis B, liver cirrhosis, hepatocellular carcinoma [2-4]. Previous studies showed the host genetic factor played a critical role in HBV infection susceptibility and identified associated SNPs with significant contribution, including major histocompatibility complex (MHC) genes, i.e. HLA-DPA1 (rs3077), HLA-DPB1 (rs9277535), HLA-C (rs3130542), HLA-DQ (rs2856718, rs7453920) [5-7], and non-MHC genes, i.e. UBE2L3 (rs4821116), INTS10 (rs7000921) [8, 9]. In advanced stages of HBV disease, host genetic factors influence the outcome of HBV infection [7, 10, 11], including $H L A$ $D Q$ (rs9275319), HLA-DRB1 (rs2647073, rs3997872), STAT4 (rs7574865), C2 (rs9267673), PNPLA3 (rs738408, rs738409), SLC17A2 (rs80215559), HFE (rs1800562) [12, 13] for liver cirrhosis and KIF1B (rs17401966), HLA$D Q A 1 / D R B 1$ (rs9272105), HLA-DQ (rs9275319), STAT4 (rs7574865) for hepatocellular carcinoma [14-16]. However, these reported HBV-related genes confer relatively small increments in risk and explain a small proportion of heritability. For example, although MHC genes are important for immune response to $\mathrm{HBsAg}$, more than half the heritability is determined by non-MHC genes [17]. Moreover, previous studies showed that the MHC genes share a common influence on HBV infection, liver cirrhosis, hepatocellular carcinoma $[6,12,15,16]$ as well as associate with different risk in these outcomes [18]; i.e. HLA-DQ, STAT4, C2, HLA-DRB1 for liver cirrhosis and HCC [12], HLA-DQ for CHB [6]. These consistent [12] or different [18] risks indicated shared but also modified effects for progressive HBV-related outcomes. These results raised our interest to identify host genetic factor which increases the risk of progressive stages post HBV infection. To reveal new susceptibility genes for HBV infection and the HBV-related outcomes, we performed a genome-wide association study (GWAS) in 1031 participants, including 275 HBV clearance subjects, 92 asymptomatic persistence infection carriers (ASPI), 93 chronic hepatitis B patients (CHB), 188 HBV-related decompensated cirrhosis patients (DC), 214 HBV-related hepatocellular carcinoma patients (HCC) and 169 healthy controls (HC) (Table 1).

Table 1 Characteristics of participants in the genome-wide association cohorts

\begin{tabular}{|c|c|c|c|c|c|c|}
\hline Disease categories & HBV clearance & ASPI & $\mathrm{CHB}$ & DC & $\mathrm{HCC}$ & $\mathrm{HC}$ \\
\hline Sample size & 275 & 92 & 93 & 188 & 214 & 169 \\
\hline Mean age $\pm S D$ & $49.56 \pm 8.8$ & $46.89 \pm 6.9 *$ & $46.46 \pm 5.7^{* *}$ & $50.65 \pm 8.4$ & $51.34 \pm 10.5 *$ & $48.82 \pm 7.1$ \\
\hline Male/female & $105 / 170$ & $33 / 59$ & $62 / 31 * * *$ & $148 / 40 * * *$ & $183 / 31 * * *$ & $73 / 96$ \\
\hline $\mathrm{ALT}, \mathrm{U} / \mathrm{L}$, mean $\pm \mathrm{SD}$ & $26.95 \pm 30.42$ & $24.5 \pm 8.79$ & $169.53 \pm 243.76^{* * *}$ & $89.57 \pm 115.79$ *** & $214 \pm 381.67^{* *}$ & $23.11 \pm 7.97$ \\
\hline $\mathrm{AST}, \mathrm{U} / \mathrm{L}$, mean $\pm \mathrm{SD}$ & $25.89 \pm 21.89 *$ & $23.16 \pm 6.45$ & $100.04 \pm 127.54^{* * *}$ & $102.46 \pm 118.36^{* * *}$ & $120.51 \pm 286.05 * * *$ & $22.23 \pm 7.00$ \\
\hline $\mathrm{TBiL}, \mu \mathrm{mol} / \mathrm{L}$, mean $\pm \mathrm{SD}$ & $13.68 \pm 9.93$ & $12.28 \pm 2.91$ & $23.12 \pm 35.60^{* * *}$ & $65.87 \pm 83.22^{* * *}$ & $50.11 \pm 90.93^{* * *}$ & $12.95 \pm 4.09$ \\
\hline $\mathrm{DBiL}, \mu \mathrm{mol} / \mathrm{L}$, mean $\pm \mathrm{SD}$ & $5.86 \pm 18.39$ & $3.59 \pm 1.68$ & $9.36 \pm 14.29 * *$ & $35.15 \pm 51.33^{* * *}$ & $25.87 \pm 55.04 * * *$ & $4.54 \pm 9.46$ \\
\hline $\mathrm{ALP}, \mathrm{U} / \mathrm{L}$, mean $\pm \mathrm{SD}$ & $74.30 \pm 35.86$ & $73.57 \pm 44.58$ & $108.89 \pm 42.20 * * *$ & $132.22 \pm 61.42^{* * *}$ & $141.63 \pm 104.32 * * *$ & $70.85 \pm 25.33$ \\
\hline $\mathrm{GGT}, \mathrm{U} / \mathrm{L}$, mean $\pm \mathrm{SD}$ & $28.34 \pm 30.77$ & $29.88 \pm 21.19$ & $88.76 \pm 106.63 * * *$ & $89.94 \pm 132.10 * * *$ & $158.08 \pm 183.92 * * *$ & $27.58 \pm 24.88$ \\
\hline$A L B, g / L$, mean $\pm S D$ & $43.33 \pm 6.11$ & $44.16 \pm 6.71$ & $42.40 \pm 42.25 *$ & $32.28 \pm 7.13^{* *}$ & $37.19 \pm 7.27^{* * *}$ & $43.93 \pm 4.96$ \\
\hline AFP, $\mu \mathrm{g} / \mathrm{L}$, mean $\pm S D$ & $21.39 \pm 47.39 *$ & $5.16 \pm 4.79$ & $34.00 \pm 89.37^{*}$ & $87.74 \pm 169.83 * *$ & $7315.22 \pm 37,329.94$ & $3.76 \pm 4.34$ \\
\hline PTA, \%, mean $\pm S D$ & $95.22 \pm 52.69$ & $91.67 \pm 14.30$ & $90.06 \pm 21.76$ & $63.97 \pm 28.07 * * *$ & $87.31 \pm 33.96$ & $90.84 \pm 18.71$ \\
\hline $\mathrm{PLT}, 10^{9} / \mathrm{L}$, mean $\pm \mathrm{SD}$ & $147.69 \pm 55.40$ & $146.84 \pm 44.51$ & $150.27 \pm 49.61$ & $73.96 \pm 45.30 * * *$ & $145.72 \pm 79.24$ & $148.94 \pm 49.10$ \\
\hline
\end{tabular}

Abbreviations: $\mathrm{ASPI}$, asymptomatic persistence infection; $\mathrm{CHB}$, chronic hepatitis $\mathrm{B}$; $\mathrm{DC}$, decompensated cirrhosis; $\mathrm{HCC}$, hepatocellular carcinoma; $\mathrm{HC}$, healthy controls; SD, standard deviation; ALT, alanine aminotransferase; AST, aspartate aminotransferase; TBIL, total bilirubin; DBIL, direct bilirubin; ALP, alkaline phosphatase; GGT, glutamyl transpeptidase

Notes: ${ }^{*}$, statistical significance of the difference between each case group and HC group $\left.{ }^{* * *}: p=[0,0.001], * *: p=(0.001,0.01],{ }^{*}: p=(0.01,0.05]\right)$. The significances of gender and other characteristics were calculated by Fisher's exact test and ANOVA test, respectively 


\section{Methods}

\section{Study participants}

A total of 1104 unrelated, age- and gender- matched, Chinese participants were recruited in the study, enrollment criteria were consistent with a previous report [19]. The population of HBV-related phenotypes was composed of five subgroups: HBV clearance subjects, asymptomatic persistence infection (ASPI) carriers, chronic hepatitis $\mathrm{B}(\mathrm{CHB})$ patients, HBV-related decompensated cirrhosis (DC) patients, HBV-related hepatocellular carcinoma (HCC) patients. Healthy controls (HC) who were $\mathrm{HBV}$ serum marker-negative (HBsAg, anti-HBc) and had no serological evidence of co-infection with HCV, HDV, and HIV were also included. HBV chronic infection patients were diagnosed based on seropositivity of HBsAg at least 6 months. Then ASPI was defined as HBsAg and anti-HBc positive at least 6 months and serum alanine aminotransferase (ALT), aspartate aminotransferase (AST) in normal values without abnormal before. $\mathrm{CHB}$ is defined as $\mathrm{HBsAg}$ and anti-HBc positive at least 6 months and ALT, AST abnormal before or at enrollment. DC was defined as HBsAg and anti-HBc positive at least 6 months with decompensated portal hypertension (gastroesophageal bleeding, ascites, edema or encephalopathy) or decompensated liver function (albu$\min <35 \mathrm{~g} / \mathrm{L}$ and total bilirubin $>35 \mathrm{umol} / \mathrm{L}$ ). HCC was defined at least one of following: (a) liver biopsy; or (b) abnormal alpha fetoprotein (AFP) and sonographic, CT or MRI space occupying evidence.

\section{Clinical parameters}

Clinical parameters including serum alanine aminotransferase (ALT), aspartate aminotransferase (AST), total bilirubin (TBIL), direct bilirubin (DBIL), alkaline phosphatase (ALP), glutamyl transpeptidase (GGT), albumin (ALB), globulin (Glo), alpha fetoprotein (AFP), prothrombin time activity (PTA), platelets (PLT), HBsAg, anti-HBs, HBeAg, anti-HBe, anti-HBc were collected from hospital information system. Other baseline characteristics were recorded during each patient's clinical examination. In brief, liver biochemistry and virological tests were carried out by Bechman Coulter AU chemistry analyzers, chemiluminescence immunoassays (AxSYM or ARCHITECT I2000, Abbott, USA) or Ortho/Chemiluminescent assay (Johnson and Johnson Co., USA) with commercially available kits; Anti-HAV IgM antibody, HDV antigen (HDAg) and anti-HDV antibody, and antiHEV antibody were determined by commercially ELISA kits in China. For HBV DNA level, it was quantified using commercial real-time polymerase chain reaction kit with a lower limit of detection (LLOD) of $100 \mathrm{IU} / \mathrm{ml}$ (Daan company, China) or Roche Cobas Ampliprep/Cobas
Taqman $^{\mathrm{TM}}$ PCR assay with LLOD of $20 \mathrm{IU} / \mathrm{ml}$ (Roche, USA).

\section{Genome-wide SNP genotyping and quality control}

Genotyping was performed on Affymetrix 500k Genome-Wide Human SNP Array 6.0 (http://www.affym etrix.com/Auth/analysis/downloads/na35/genotyping/ GenomeWideSNP_6.na35.annot.csv.zip). SNPs met the following quality control procedures were excluded: (1) call rate $<95 \%$; (2) minor allele frequency (MAF) $<1 \%$; (3) genotype in controls deviated from the Hardy Weinberg equilibrium (HWE test $\mathrm{P}$-value $<10^{-5}$ ).

\section{Statistics analysis}

GCTA tool [20] was used to perform principal component analyses for estimating population substructure. The first two eigenvectors, pc1 and pc2, were used to display the population structure. PLINK 1.9 [21] software was used to perform logistic regression for identifying susceptibility SNPs of HBV infection and HBV-related outcomes. Gender and age were used as covariates in logistic regression. Chi-square test for trend in proportions was used to identify SNPs with increased effectiveness on disease progression. We used the Bonferroni method to adjust the false positive rate caused by multiple test. The number of independent LD block was used to represent the number of independent multiple test. We calculated a total of 21,077 independent LD blocks via GEC [22] and then set $0.05 / 21077$ as the threshold of genome-wide significance. The genomic control method was used to measure population stratification by calculating the genomic inflation factor $(\boldsymbol{\lambda})$ from median P-value. ANOVA was used to evaluate the significance of the association between biomarkers and genotypes in healthy controls. Using the SNPs in HBV infection-related loci in 1000 Genomes Project [23], we performed evolutional analyses, including building phylogenetic tree, detecting the signatures of selection, displaying the core haplotypes, estimating effective population size. Derived allele and ancestral allele of SNPs were accessed from Ensemble human ancestral genome (http://ftp.1000genomes.ebi.ac.uk/vol1/ftp/phase1/analy sis_results/supporting/ancestral_alignments). PoMo [24], an allele frequency-based approach, was used to build the racial tree based on the allele frequency of SNPs in each population. $\mathrm{F}_{\mathrm{ST}}$ [25], a classical metrics of population differentiation, was widely employed in detecting signatures of selection [26] in human genome [27, 28] and animal genome [29-31]. In our study, $\mathrm{F}_{\mathrm{ST}}$ was implemented to detect the selective signature between East Asian population and each other population. Vcftools [32] was used to calculate the $\mathrm{F}_{\mathrm{ST}}$ statistics of SNPs in paired populations. $\mathrm{F}_{\mathrm{ST}}$ statistics accesses 0.15 [33] was used as a threshold to 


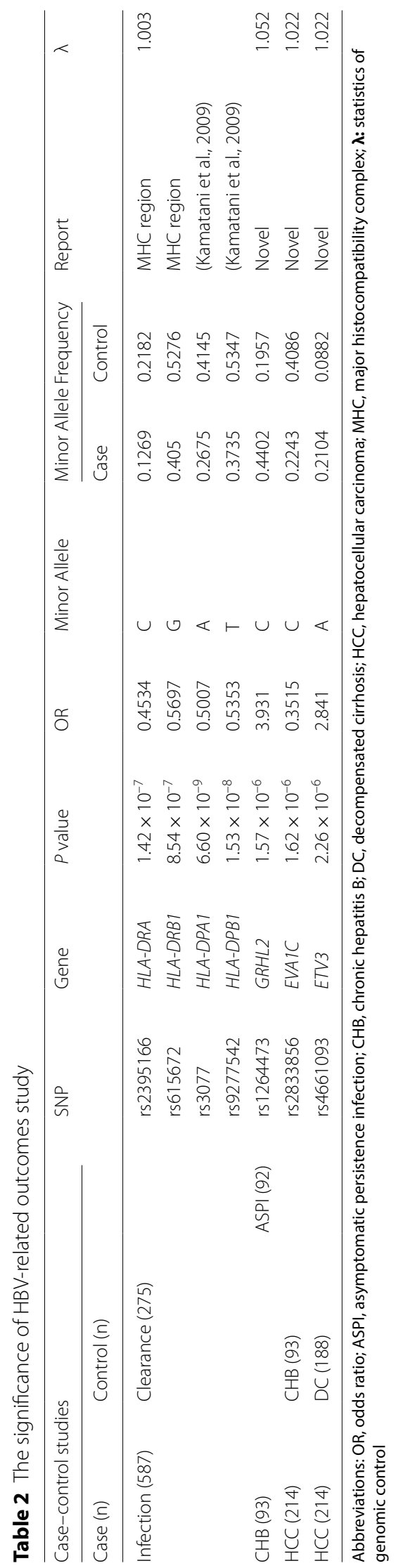


detect the signature of selection. Rehh package [34, 35] was used to display the haplotype bifurcation diagrams of the associated SNPs in different populations. Relate [36], a method for genome-wide genealogy estimation for thousands of samples, was used to estimate the historical population size at default setting.

\section{Results}

There are 1031 participants passed quality control from 1104 participants. The demographic and clinical characteristics of 1031 study participants included in our association study are presented in Table 1 . All participants were genotyped by Affymetrix 500k SNP Array. A total of 607,153 SNPs passed through quality control (Additional file 1: Figure S1). These SNPs filtered minor allele frequency of $<1 \%$ and a call rate of $<95 \%$.

To demonstrate that there is no genetic stratification in the population, we performed a principal component analysis on the SNPs of all participants. The first two principal components show absence of population structure (Additional file 1: Figure S2). To identify susceptibility SNPs for HBV infection, we performed a GWAS in HBV infection similar with previous design $[8,9]$. HBV clearance was used as a control group versus ASPI, CHB, DC, HCC as HBV chronic infection (case group). We observed associations of two novel MHC loci with progression to certain HBV stages (SNP: rs2395166, Gene: HLA-DRA, $P=1.42 \times 10^{-7}$; SNP: rs615672, Gene: $H L A$ DRB1, $P=8.54 \times 10^{-7}$ ) and two reported MHC loci (SNP: rs3077, Gene: HLA-DPA1, $P=6.60 \times 10^{-9}$; SNP: rs9277542, Gene: $H L A-D P B 1, P=1.53 \times 10^{-8}$ ) (Table 2; Fig. 1). These MHC loci variants replicated association results of previous studies affirming that MHC gene alleles confer risks of susceptibility of HBV infection in East Asian. Interestingly, we found that these reported MHC loci (rs2395166:C, rs615672:G, rs3077:A, rs9277542:T, rs9277341:T) present significant differences in allele frequency between East Asian and nonEast Asian population in gnomAD database (Table 3), as well as the differences between HBV infection group and HBV clearance group. Since different groups may not present an identical minor allele, here, we used the derived allele against the ancestral allele for studying the allele frequency across different populations. The derived allele frequencies in East Asian are much closer to the HBV chronic infection group, while other populations, such as European, are much closer to the HBV clearance group. These genetic differences may suggest a selective signal in non-East Asian population versus East Asian population. To confirm this, we firstly build a phylogenetic tree based on these loci and then showed the genetic diversity in world-wide populations, in which the East Asian population is at the root. We set the East
Asian as the ancestral group in these loci according to the derived allele frequencies and the phylogenetic tree. Subsequently, we identified two strong phylogenetic signals (HLA-DPA1, HLA-DPB1) in the European population (Fig. 2) via $\mathrm{F}_{\mathrm{ST}}$ method. Haplotype bifurcation diagrams of the two core SNPs (rs3077, rs9277542) presented that the resisted allele led to a long-range, and a high frequency homozygosity in European population (Fig. 3), confirming the natural genetic selection. These evidences revealed that the resisted alleles were under positive selection in European population strongly. We estimated the historic population size and then showed these two loci (HLA-DPA1, HLA-DPB1) were under selection during the past 26,000 years (Additional file 1: Figure S3). These results may provide a context for the racking influence of HBV infectious diseases in history.

To identify new susceptibility locus for HBV-related outcomes, we performed association studies for CHB, DC, and HCC. Significantly, we observed three associated gene SNP loci: (1) (SNP: rs1264473, Gene: GRHL2, $P=1.57 \times 10^{-6}$ ) associated with $\mathrm{CHB}$ versus ASPI; (2) (SNP: rs2833856, Gene: EVA1C, $P=1.62 \times 10^{-6}$ ) associated with HCC versus CHB; and (3) (SNP: rs4661093, Gene: $E T V 3, P=2.26 \times 10^{-6}$ ) associated with $\mathrm{HCC}$ versus DC (Table 2; Fig. 1). No SNP associated with DC versus CHB were apparent.

HBV clearance, ASPI, CHB, DC, and HCC are progressive stages post HBV infection [4]. We hypothesized that the host genetic factor contributes to the development of outcomes, as well as to the individual outcome. To investigate this hypothesis, we test two progressive stages upon HBV infection: 1.) HBV infection itself (CHB, ASPI, and HBV clearance) and 2.) development of $\mathrm{CHB}$ (CHB, DC, and HCC). We performed a chisquare test for trend in proportions of allele to identify SNPs increasing risk of HBV-related outcomes in the progressive stages. We observed association with one novel locus (SNP: rs1537862, Gene: LACE1, $\left.P=1.85 \times 10^{-6}\right)$, one association with a reported locus (SNP: rs9277542, Gene: $H L A-D P B 1, P=1.50 \times 10^{-9}$ ), and two association variants at MHC genes (SNP: rs615672, Gene: HLA-DRB1, $P=1.39 \times 10^{-6}$; SNP: rs3128923, Gene: $H L A-D P A 2, P=2.06 \times 10^{-6}$ ) with trend test of allele frequency across three outcomes (Table 4; Fig. 4A). The three reported MHC genes were demonstrated to play a critical role in the resistance of HBV infection, and two (HLA-DPB:rs9277542, $H L A-D R B 1$ :rs9277542) were identified to be associated with HBV clearance (Table 2). We did not observe any SNPs achieve genome-wide significant association with development of CHB; One additional locus (SNP: 


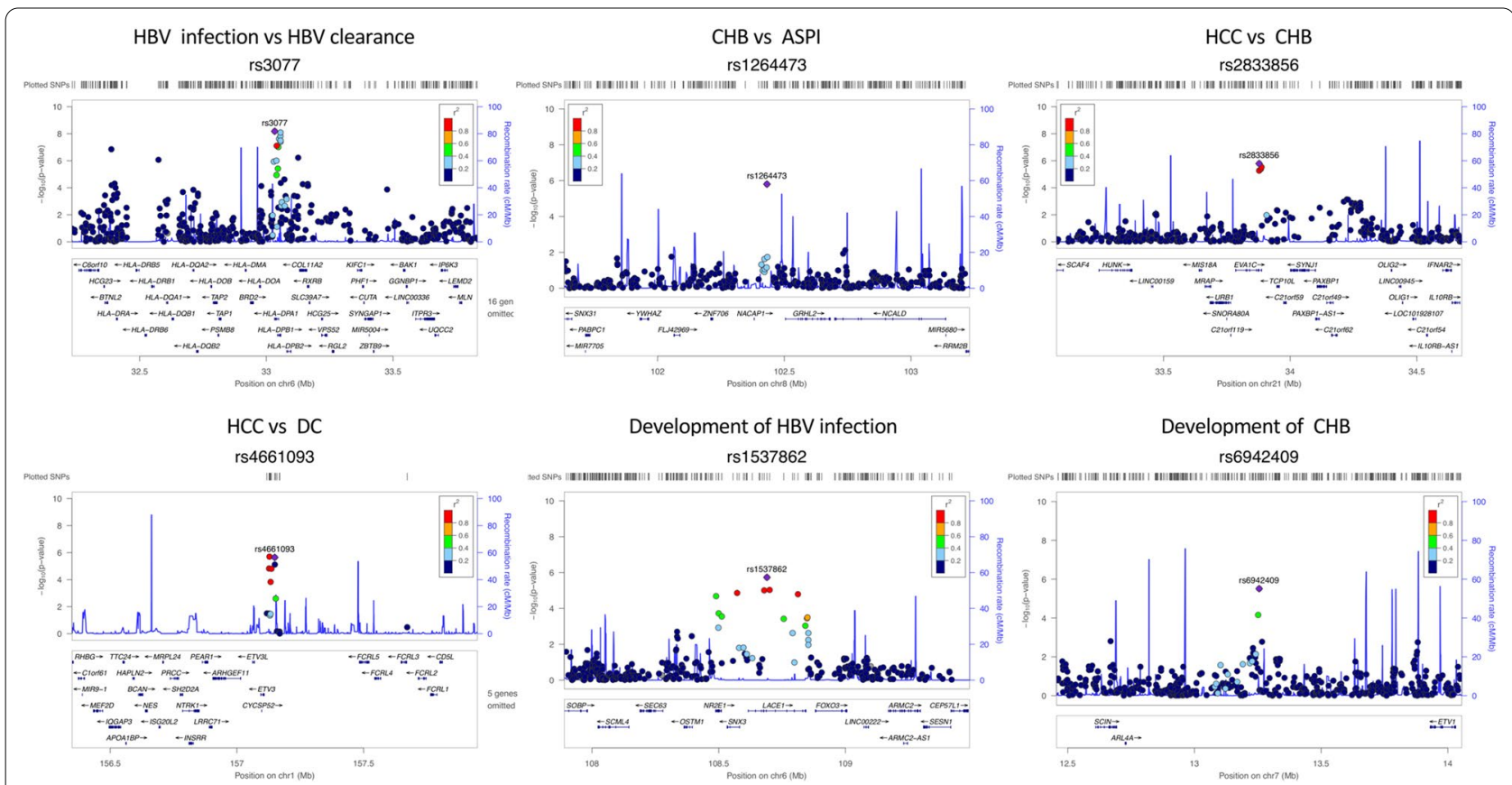

Fig. 1 Regional plots shown -log10 P-values of SNPs in association study. Marker SNPs are shown as purple diamonds, other SNPs are shown as dots. R-square of Marker SNPs and other SNPs are shown against dark blue, blue, green, yellow and red colors, indicating the linkage disequilibrium. The structure of genes within the region are shown as rectangles and arrows. Abbreviation: ASPI, asymptomatic persistence infection; $\mathrm{CHB}$, chronic hepatitis B; DC, decompensated cirrhosis; HCC, hepatocellular carcinoma

Table 3 Divided allele frequency of significant SNPs in MHC region

\begin{tabular}{|c|c|c|c|c|c|c|c|c|c|}
\hline \multirow[t]{3}{*}{ SNP } & \multirow[t]{3}{*}{ Study } & \multirow[t]{3}{*}{ Population } & \multirow{3}{*}{$\begin{array}{l}\text { Derived } \\
\text { Allele }\end{array}$} & \multicolumn{5}{|c|}{ Derived Allele Frequency } & \multirow{3}{*}{$\begin{array}{l}P \text { value } \\
\text { East Asian vs } \\
\text { non-East Asian }\end{array}$} \\
\hline & & & & \multicolumn{3}{|c|}{ Case-control studies } & \multicolumn{2}{|l|}{ gnomAD } & \\
\hline & & & & Infection & Clearance & Healthy & East Asian & non-East Asian & \\
\hline rs2395166 & our study & Chinese & C & 0.127 & 0.218 & 0.183 & 0.128 & 0.367 & $4.367 \times 10^{-195}$ \\
\hline rs615672 & our study & Chinese & G & 0.405 & 0.528 & 0.512 & 0.388 & 0.590 & $6.003 \times 10^{-111}$ \\
\hline \multirow[t]{5}{*}{ rs3077 } & our study & Chinese & A & 0.268 & 0.415 & 0.379 & 0.283 & 0.723 & \\
\hline & Kamatani et al., 2009 [5] & Japanese & & 0.245 & - & 0.392 & & & \\
\hline & Guo et al., 2011 [55] & Chinese & & 0.314 & 0.447 & 0.443 & & & 0 \\
\hline & Nishida et al., 2012 [56] & Japanese, Korean & & 0.213 & 0.393 & - & & & \\
\hline & Wong et al., 2013 [57] & Southern Chinese & & 0.206 & 0.276 & 0.288 & & & \\
\hline \multirow[t]{2}{*}{ rs9277341 } & our study & Chinese & $\mathrm{T}$ & 0.142 & 0.242 & 0.196 & 0.159 & 0.582 & 0 \\
\hline & Guo et al., 2011 [55] & Chinese & & 0.133 & 0.237 & 0.237 & & & \\
\hline \multirow[t]{2}{*}{ rs9277542 } & our study & Chinese & $\mathrm{T}$ & 0.374 & 0.535 & 0.482 & 0.339 & 0.627 & $2.005 \times 10^{-225}$ \\
\hline & Kamatani et al., 2009 [5] & Japanese & & 0.246 & - & 0.437 & & & \\
\hline
\end{tabular}

Abbreviations: East Asian, East Asian population in gnomAD database; non-East Asian, combined all other population excepted East Asian in gnomAD database; $P$ value, compared allele frequency between East Asian and non-East Asian population via fisher exact test. Derived Allele was accessed from human ancestral genome (Ensembl-59)

rs6942409, Gene: $\left.A C 011288.2, P=3.08 \times 10^{-6}\right)$ and the HCC associated locus (SNP: rs2833856, Gene: EVA1C, $P=1.62 \times 10^{-5}$ ) were associated with increased risk of $\mathrm{DC}$ and $\mathrm{HCC}$ during the development of $\mathrm{CHB}$ (Table 5; Fig. 4 b).
Host genetic factors were demonstrated to influence concentrations of liver enzymes in plasma, which are widely used to indicate liver disease [37, 38]. Here, to investigate the functional change in liver influenced by the HBV related loci described above, we performed 

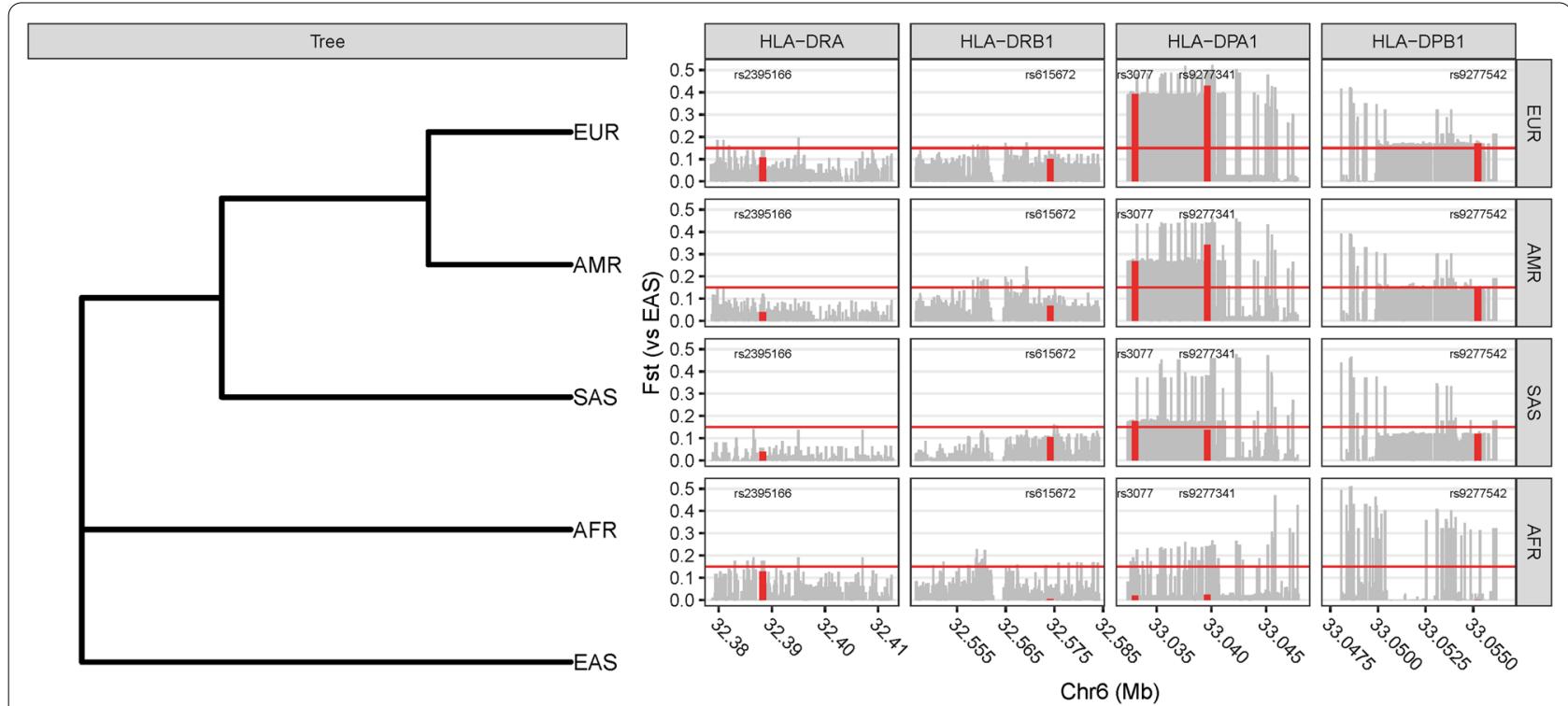

Fig. 2 The racial tree (Left) was based on the SNPs in HBV-infection related genes, including HLA-DRA, HLA-DRB1, HLA-DPA1 and HLA-DPB1. The genotype and minor allele frequency of each SNPs were accessed from 1000 Genome Project. EAS, AFR, SAS, EUR, AMR refer to East Asian, African, South Asian, European and American of 1000 Genome Project, respectively. The racial tree indicated a genetic difference in HBV-infection related genes among EAS, AFR, SAS, EUR, AMR. The genetic difference (Right) of each SNPS was evaluated by $F_{S T}$ value. X-axis refer to physical position in chromosome 6. Y-axis refer to $F_{S T}$ value of paired SNP. $F_{S T}$ values of all paired SNPS of AFR, SAS, AMR, EUR versus EAS were displayed in grey bar. $F_{S T}$ values accessed 0.15 (Red horizontal line) indicated the signal of selective event. Red bars and rs IDs showed the reported HBV infection-related SNPs. The $F_{S T}$ values of European versus East Asian showed the genetic difference in HLA-DPA1 and HLA-DPB1, indicating a genetic selection against the HBV infection. The racial tree showed that the East Asian population is at the root, indicating that why we used East Asian population as a comparative population but not the other population, and compared other four populations with East Asian population

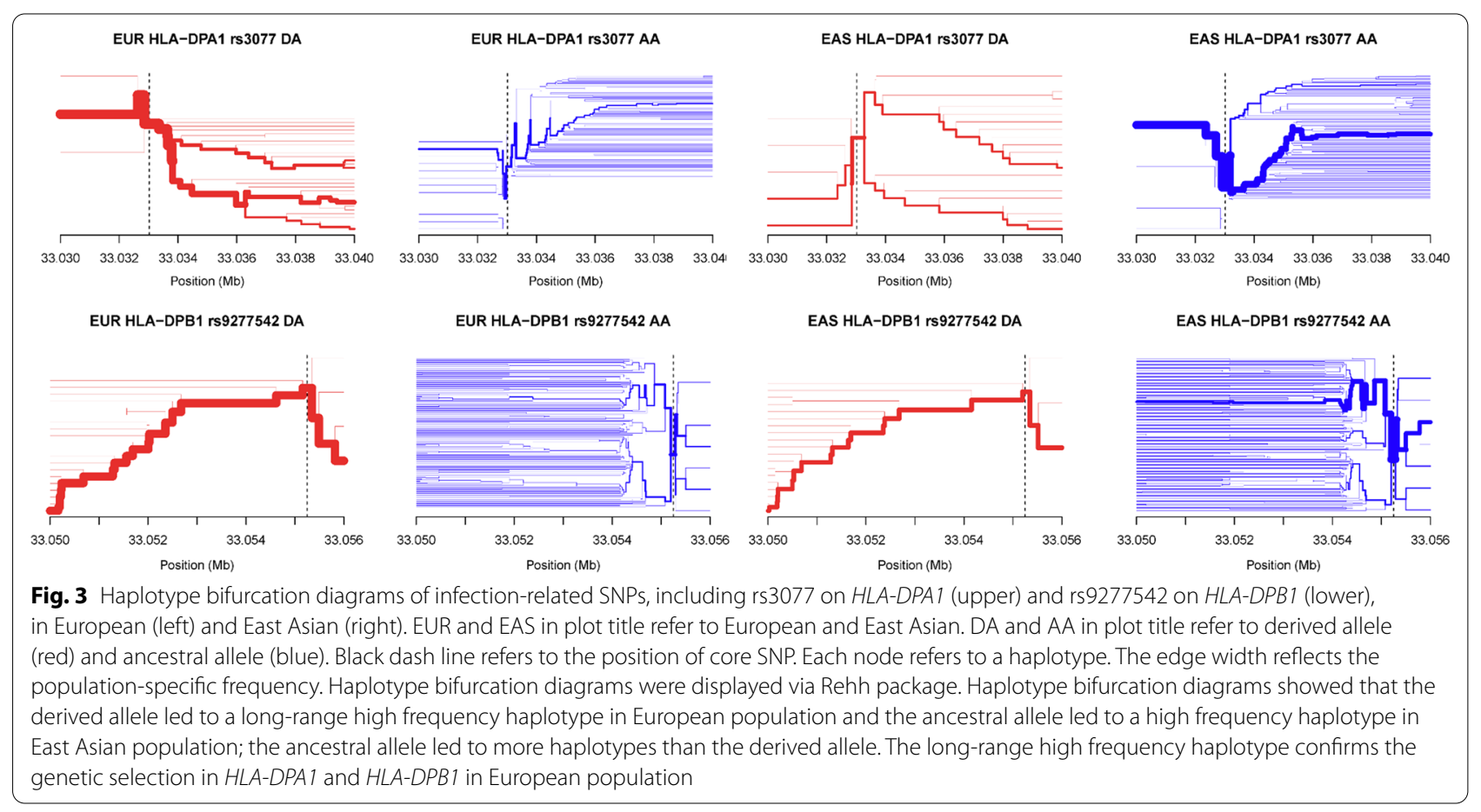


Table 4 The significance of progressive HBV infection study

\begin{tabular}{|c|c|c|c|c|c|c|c|c|}
\hline \multirow[t]{2}{*}{ SNP } & \multirow[t]{2}{*}{ Gene } & \multirow[t]{2}{*}{$P$ value } & \multirow[t]{2}{*}{ Resistant Allele } & \multicolumn{3}{|c|}{ Resistant Allele Frequency } & \multicolumn{2}{|l|}{ Related Risk } \\
\hline & & & & $\mathrm{CHB}$ & ASPI & Clearance & CHB (vs ASPI) & $\begin{array}{l}\text { Clearance } \\
\text { (vs ASPI) }\end{array}$ \\
\hline rs615672 & HLA-DRB1 & $1.39 \times 10^{-6}$ & G & 0.3297 & 0.4185 & 0.5276 & 0.82 & 1.12 \\
\hline rs9277542 & HLA-DPB1 & $1.50 \times 10^{-9}$ & $\mathrm{~T}$ & 0.3011 & 0.375 & 0.5347 & 0.84 & 1.17 \\
\hline rs3128923 & HLA-DPA2 & $2.06 \times 10^{-6}$ & G & 0.3804 & 0.4348 & 0.5636 & 0.89 & 1.14 \\
\hline rs1537862 & LACE1 & $1.85 \times 10^{-6}$ & C & 0.6 & 0.6374 & 0.7647 & 0.92 & 1.19 \\
\hline
\end{tabular}

Abbreviations: ASPI, asymptomatic persistence infection; $\mathrm{CHB}$, chronic hepatitis B. RR was calculated with the comparison of CHB and ASPI, Clearance and ASPI respectively
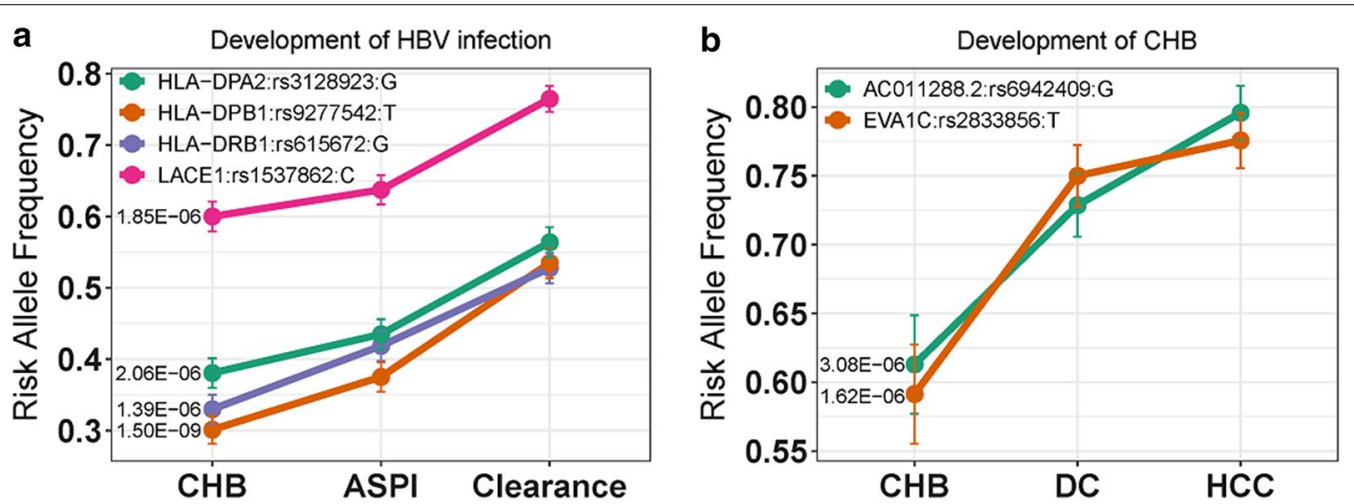

Fig. 4 The raising allele frequency in HBV related outcomes during the progression. Four SNPs with increased resistance in $\mathrm{CHB}, \mathrm{ASPI}, \mathrm{HBV}$ clearance during HBV infection (a) and two SNPs with increased risk in the CHB, DC, HCC during the development of CHB (b). Abbreviation: ASPI, asymptomatic persistence infection; CHB, chronic hepatitis B; DC, decompensated cirrhosis; $H C C$, hepatocellular carcinoma

Table 5 The suggestive significance of progressive CHB study

\begin{tabular}{|c|c|c|c|c|c|c|c|c|}
\hline \multirow[t]{2}{*}{ SNP } & \multirow[t]{2}{*}{ Gene } & \multirow[t]{2}{*}{$P$ value } & \multirow[t]{2}{*}{ Risk Allele } & \multicolumn{3}{|c|}{ Risk Allele Frequency } & \multicolumn{2}{|l|}{ Related Risk } \\
\hline & & & & $\mathrm{CHB}$ & DC & $\mathrm{HCC}$ & $\mathrm{DC}$ (vs CHB) & $\mathrm{HCC}$ (vs CHB) \\
\hline rs6942409 & AC011288.2 & $3.08 \times 10^{-6}$ & G & 0.6129 & 0.7287 & 0.7958 & 1.20 & 1.37 \\
\hline rs2833856 & EVATC & $1.62 \times 10^{-5}$ & $\mathrm{~T}$ & 0.5914 & 0.75 & 0.7757 & 1.30 & 1.35 \\
\hline
\end{tabular}

Abbreviations: $\mathrm{CHB}$, chronic hepatitis B; DC, decompensated cirrhosis; $\mathrm{HCC}$, hepatocellular carcinoma. RR was calculated with the comparison of $\mathrm{HCC}$ and $\mathrm{CHB}, \mathrm{DC}$ and $\mathrm{CHB}$ respectively

a variance analysis in 10 clinical parameters of serum liver enzymes (ALT, AST, TBIL, DBIL, ALP, GGT, ALB, AFP, PTA, and PLT) between different genotypes in healthy controls (Additional file 1: Figure S4-9). Six loci (rs1537862, rs3128923, rs9277542, rs9277341, rs9277378, rs4661093) showed modest associations with concentrations of liver enzymes, including ALB, ALP, AFP, and PTA (Fig. 5). These associations suggest pathways linking the host genetic factors, metabolism, and liver function for understanding the mechanisms of infection and disease progression.

In sum, our study identified susceptibility SNPs associated with HBV related outcomes and SNPs increased the risk of progressive outcomes from HBV clearance to
HBV chronic infection, DC, and HCC in a Chinese population (Additional file 1: Figure S10).

\section{Discussion}

HBV infection leads to a wide spectrum of clinical outcomes, including spontaneous clearance, asymptomatic carrier, chronic hepatitis B, liver cirrhosis, and hepatocellular carcinoma. Previous studies showed that MHC genes played an important role in outcomes of HBV infection [7]. Alleles associated with HBV infection versus HBV clearance affect infection risk, and a low-risk allele indicated an effect on virus clearance. By contrast loci associated with $\mathrm{CHB}$ versus ASPI indicated risk for 

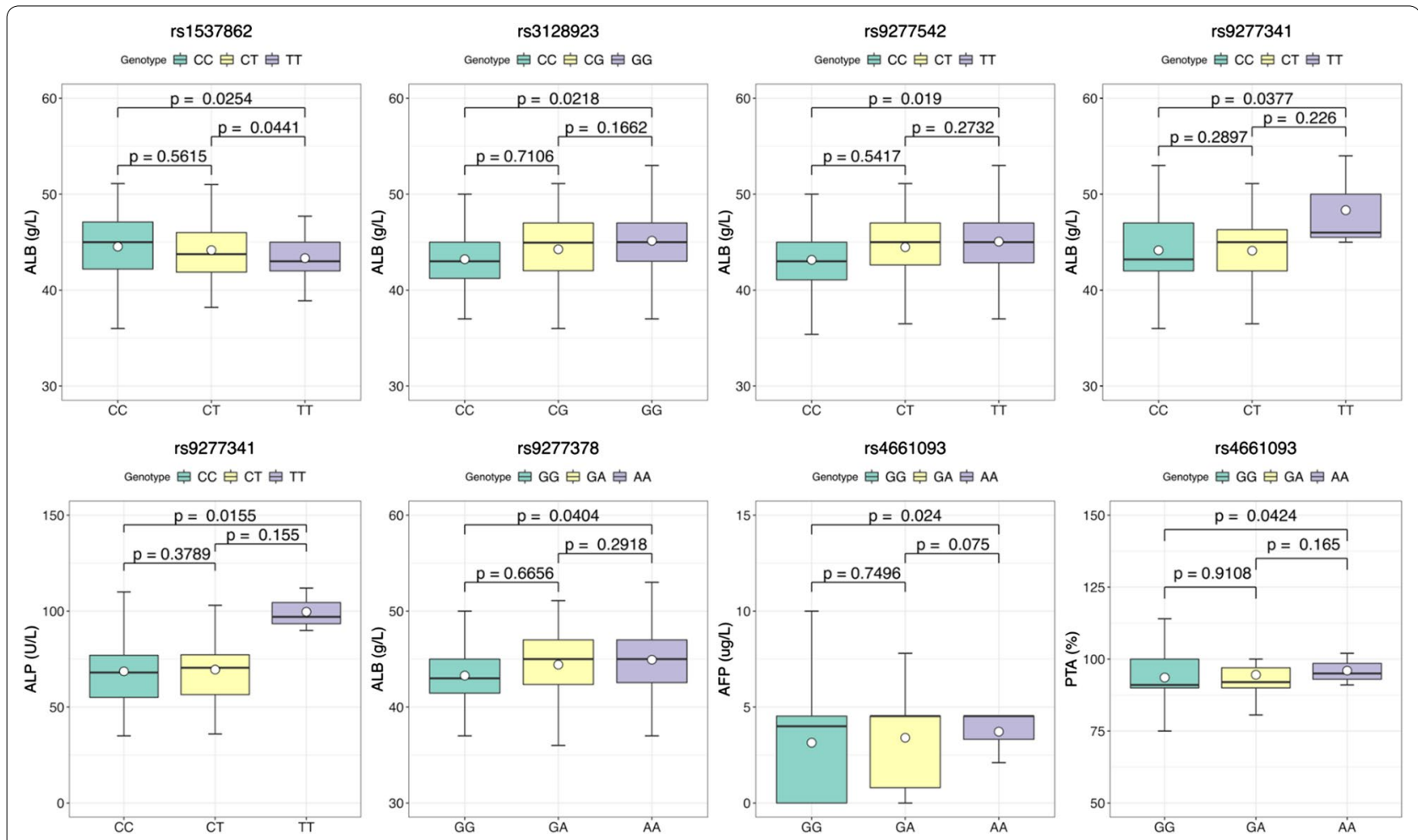

Fig. 5 The association between HBV related loci and serum liver enzyme levels in health controls. $P$ values were calculated by ANOVA test. White-circle refer to the mean liver enzymes level with different genotypes. The significant differences indicate that these SNPs contribute to liver enzyme activity

the severe progression, while a low-risk allele affected tolerance of virus. The tolerance-related gene, GRHL2, was demonstrated to influence the inflammation in hepatocytes by regulating microRNA 122 (MIR122) and the target of MIR122, HIF $1 \alpha$ [39]. Levels of GRHL2 were increased in liver tissues of patients with alcoholic liver disease and correlated with decreases in levels of MIR122. Increased levels of MIR122 in hepatocytes of mice with ethanol-induced liver disease and advanced fibrosis reduced levels of HIF $1 \alpha$ and reduced serum levels of alanine aminotransferase (ALT). Taken together, we propose that the low-risk allele rs1264473:T at GRHL2 ablates severe persistent inflammation through increased the levels of MIR122.

Our previous studies $[40,41]$ showed that NTCP S267F mutation significantly affected the disease progression to cirrhosis $(P=0.017)$, and hepatocellular carcinoma $(P=0.023)$ versus $\mathrm{CHB}[40]$ and the rs3077:T allele was associated with decreased risk of chronic HBV infection $(\mathrm{OR}=0.62, P=0.001)$ [41]. In this study, we searched for host genetic factor with increased risk of the development-related outcomes in GWAS. One novel locus, $L A C E 1$, and three infection-related MHC loci were associated the progression of HBV infection. These results showed that the host genetic factors, both $\mathrm{MHC}$ and non-MHC genes, increased the risk of progressive outcomes post $\mathrm{HBV}$ infection, as well as HBV mutation. It is reported that HBV infection altered the mitochondrial metabolism and mitochondrial dynamics, which result in mitochondrial injury and liver disease [42]. LACE1 was reported to affect mitochondrial protein homeostasis [43]. Knockdown of LACE1 converted the expression of a crucial component of regulating mitochondrial dynamics, OPA1 [43-45]. In addition, we found that the risk allele, $L A C E 1$ :rs1537862:T, decreased the level of ALB significantly ( $P=0.025$, Fig. 5). ALB is a critical marker decreasing with the deterioration of chronic liver diseases [46-48]. Biosynthesis of ALB was affected by proinflammatory cytokines $[49,50]$ and excess amounts of oxidative agents released by mitochondria from injured liver $[46,51]$. Taken together, we proposed $L A C E 1$ may affect hepatic infection by changing the hepatic mitochondrial metabolism and leading to the progression of $\mathrm{HBV}$ infection.

There is a limitation in our study, that is we do not have an additional cohort for replicate study. In spite of that, 
we showed the reported loci in MHC region are significantly related to HBV infection. These replicate results of previous studies confirm our findings are reliable and provide confidence for our study in this cohort. Here, we provide novel candidate genes related to individual outcomes, progressive stages, and liver enzymes. Moreover, we identified two SNPs that show selective significance (HLA-DPA1, HLA-DPB1) in non-East Asian (European, American, South Asian) versus East Asian. East Asian population seem more susceptible to HBV infection than non-East Asian, and the differences of susceptibility were affected by HBV genotype [52], immunity [53], and environmental exposure $[53,54]$. Even in an identical environment (United States), Asian are more prevalent in chronic HBV infection than non-Asian [53]. It seems likely that host genetic factors contribute to the ethnic disparities of susceptibility of HBV infection. Taken together with the genetic associations and evolutionary signals, our findings provide a new insight for HBV study.

\section{Conclusion}

In case-control study, we identified one novel locus (SNP: rs1264473, Gene: GRHL2, $P=1.57 \times 10^{-6}$ ) significantly associated with CHB, two novel loci (SNP: rs2833856, Gene: EVA1C, $P=1.62 \times 10^{-6}$; SNP: rs4661093, Gene: ETV3, $\quad P=2.26 \times 10^{-6}$ ) significantly associated with HCC. In trend study across multiple outcomes, we identified one novel locus (SNP: rs1537862, Gene: LACE1, $\left.P=1.85 \times 10^{-6}\right)$ and three MHC loci (HLA-DRB1, HLA$D P B 1, \quad H L A-D P A 2)$ significantly increased progressive risk from $\mathrm{CHB}$ through ASPI to HBV clearance. In evolutionary study, we showed the derived allele of two HBV clearance related loci, rs3077 and rs9277542, are under strong selection in European population. We suggested these selected alleles may play a role in resisting the susceptibility of HBV in Europeans. Our findings provided a new insight into the role of host genetic factors in HBV related outcomes and progression.

\footnotetext{
Abbreviations

AFP: Alpha Fetoprotein; AFR: African; ALB: Albumin; ALP: Alkaline Phosphatase; ALT: Alanine Aminotransferase; AMR: American; anti-HBC: Antibody to Hepatitis B core Antigen; anti-HBe: Antibody to Hepatitis B e Antigen; anti-HBs: Antibody to Hepatitis B surface Antigen; anti-HDV: Antibody to Hepatitis D Virus; anti-HEV: Antibody to Hepatitis E Virus; ASPI: Asymptomatic Persistence Infection; AST: Aspartate Aminotransferase; CHB: Chronic Hepatitis B; DBIL: Direct Bilirubin; DC: Decompensated Cirrhosis; EAS: East Asian; EUR: European; Glo: Globulin; GGT: Glutamyl Transpeptidase; GWAS: Genome Wide Association Study; HBeAg: Hepatitis B e Antigen; HBsAg: Hepatitis B surface Antigen; HBV: Hepatitis B Virus; HCC: Hepatocellular Carcinoma; HC: Healthy Controls; HCV: Hepatitis C Virus; HDV: Hepatitis D Virus; HIV: Human Immunodeficiency Virus; HWE: Hardy-Weinberg Equilibrium; MAF: Minor Allele Frequency; MHC: Major Histocompatibility Complex; PI: Persistence Infection; PLT: Platelets; PTA: Prothrombin Time Activity; SAS: South Asian; SD: Standard Deviation; SNP: Single Nucleotide Polymorphism; TBIL:Total Bilirubin.
}

\section{Supplementary information}

The online version contains supplementary material available at https://doi. org/10.1186/s12920-021-00907-0.

Additional file 1: Figure S1. The summary of final SNPS characteristic, including MAF, call rate, and p-value of Hardy-Weinberg equilibrium test. Figure S2. Principal component analyses indicated there are no population stratification among 6 subgroups. Abbreviation: ASPI, asymptomatic persistence infection; $\mathrm{CHB}$, chronic hepatitis $\mathrm{B}$; DC, decompensated cirrhosis; HC: healthy controls; HCC, hepatocellular carcinoma. Figure S3. Effective population sizes inferred using Related-package across all individuals of each population in two loci (HLA-DPA1, HLA-DPB1). Recentsize histories (26000 years ago) in European (purple) population showed modest difference compared with East Asian (red) population. Abbreviation: EUR, European; AMR, American; SAS, South Asian; AFR, African. Figure S4. Boxplots of rs2395166 genotype and serum liver enzyme levels in HC. Figure S5. Boxplots of rs615672 genotype and serum liver enzyme levels in HC. Figure S6. Boxplots of rs3077 genotype and serum liver enzyme levels in HC. Figure S7. Boxplots of rs 1264473 genotype and serum liver enzyme levels in HC. Figure S8. Boxplots of rs2833856 genotype and serum liver enzyme levels in HC. Figure S9. Boxplots of rs6942409 genotype and serum liver enzyme levels in HC. Figure $\mathbf{S 1 0}$. The summary of associated SNPs contributed to HBV-related outcomes and the progression. Abbreviation: PI, persistence infection; ASPI, asymptomatic persistence infection; CHB, chronic hepatitis B; DC, decompensated cirrhosis; HCC, hepatocellular carcinoma.

\section{Acknowledgements}

We thank the participants for their involvement in this study.

HBV study consortium: Department of Infectious Diseases, Peking University First Hospital, Beijing, China (Zheng Zeng, Yanyan Yu, Xiaoyuan Xu, Haiying Lu); Institute of Liver Diseases Research, Beijing Military General Hospital, Beijing, China (Darong Hu); Beijing Ditan Hospital (Rongbing Wang, Yifan Chen); Department of Surgery, Beijing Institute of Tumor Prevention and Therapy, Beijing, China (Cunyi Hao); Department of Infectious Diseases, Shanxi Medical University, Taiyuan, China (Heping Zhou); Department of Infectious Diseases, Qinhuangdao No. 3 Hospital, Qinhuangdao, China (Zhonghou Han); Department of Surgery, Inner Mongolia Medical College, Hohhot, China (Lidao Bao, Xiping Zhang); Department of Infectious Diseases, Xuzhou No. 3 Hospital, Xuzhou, China (Dasi Guo); Department of Infectious Diseases, Xinjian Medical University, Urumoqi, China (Yaoxin Zhang); Department of Infectious Diseases, the Second Affiliate Hospital of China Medical University, Shenyang, China (Xiaoguang Dou); Institute of Liver Diseases Research, Peking University People's Hospital, Beijing, China (Lai Wei); Department of Surgery, Peking Union Medical College, Beijing, China (Qiang Qu).

\section{Authors' contributions}

$Z Z, H K L, H Y L$, and HFX contributed equally. SJO and ZZ designed the study. $Z Z, H Y L, Y Y Y, X Y X, M Y, X L T, H L X$, and HBV study consortium collected samples and analyzed clinical data. ZZ and XLT maintained database. HKL, ZZ, HFX, TZ, LPG, and JGZ performed bioinformatic analyses. ZZ, SJO, and JGZ reviewed and revised the manuscript. SJO and ZZ supervised the research and made final decision. All authors have read and approves the final.

\section{Funding}

This study was supported by the National Natural Science Foundation of China (No. 61972007, No. 30671855), the International Science \& Technology Cooperation Program of China (No. 2014DFR31200), Federal funds from the National Cancer Institute, National Institutes of Health, USA (No. N01-CO-12400), Shenzhen Municipal of Government of China (JCYJ20170412153248372, JCYJ20180507183615145). These funding have no conflict of interest. The content of this publication does not necessarily reflect the view of policies of the Department of Health and Human Service, nor does mention of trade name, commercial products or organizations imply endorsement by the U.S. Government. 


\section{Availability of data and materials}

The datasets generated and/or analyzed during the current study are not publicly available as they are still being investigated but are available from the corresponding author (Dr. Zheng Zeng) on reasonable request and will be released in China National GeneBank (https://db.cngb.org/cmdb) (need to be approved by Human Genetic Resource Administration of China). The direct web links to the SNPs of 1000 Genomes Project (http://ftp.1000genomes.ebi. ac.uk/vol1/ftp/release/20130502) and Ensemble human ancestral genome (http://ftp.1000genomes.ebi.ac.uk/vol1/ftp/phase1/analysis_results/suppo rting/ancestral_alignments).

\section{Ethics approval and consent to participate}

This study was carried out following the guidelines of the 1975 Declaration of Helsinki. Informed written consent was obtained from all participants and the study was performed with the approval of the Ethics Committee of Peking University First Hospital and local cooperation hospitals (Beijing Military General Hospital, Beijing Ditan Hospital, Beijing Institute of Tumor Prevention and Therapy, Shanxi Medical University, Qinhuangdao No. 3 Hospital, Inner Mongolia Medical College, Xuzhou No. 3 Hospital, Xinjian Medical University, the Second Affiliate Hospital of China Medical University, Peking University People's Hospital, Peking Union Medical College).

\section{Consent to publish}

Not applicable.

\section{Competing interests}

The authors declared that they have no conflict of interest.

\section{Author details}

${ }^{1}$ Department of Infectious Diseases, Peking University First Hospital, Beijing 100034, China. ${ }^{2}$ BGI-Shenzhen, Shenzhen 518083, China. ${ }^{3}$ Laboratory of Genomic Diversity, Center for Computer Technologies, ITMO University, St. Petersburg, Russia 197101. ${ }^{4}$ Guy Harvey Oceanographic Center, Halmos College of Natural Sciences and Oceanography, Nova Southeastern University, Ft Lauderdale, FL 33004, USA.

Received: 3 January 2021 Accepted: 17 February 2021

Published online: 18 March 2021

\section{References}

1. Schweitzer A, et al. Estimations of worldwide prevalence of chronic hepatitis B virus infection: a systematic review of data published between 1965 and 2013. The Lancet. 2015;386(10003):1546-55.

2. Fattovich $\mathrm{G}$, et al. Occurrence of hepatocellular carcinoma and decompensation in western European patients with cirrhosis type B. Hepatology. 1995;21(1):77-82

3. Liaw Y-F, Chu C-M. Hepatitis B virus infection. The lancet. 2009;373(9663):582-92.

4. Sundaram V, Kowdley K. Management of chronic hepatitis B infection BMJ. 2015;351:h4263.

5. Kamatani $Y$, et al. A genome-wide association study identifies variants in the HLA-DP locus associated with chronic hepatitis B in Asians. Nat Genet. 2009:41(5):591.

6. Mbarek $\mathrm{H}$, et al. A genome-wide association study of chronic hepatitis B identified novel risk locus in a Japanese population. Hum Mol Genet. 2011;20(19):3884-92.

7. Zeng Z. Human genes involved in hepatitis B virus infection. World journal of gastroenterology: WJG. 2014;20(24):7696.

8. Hu Z, et al. New loci associated with chronic hepatitis B virus infection in Han Chinese. Nat Genet. 2013;45(12):1499.

9. Li Y, et al. Genome-wide association study identifies 8p21 3 associated with persistent hepatitis B virus infection among Chinese. Nature Commun. 2016;7(1):1-11.

10. Frodsham AJ. Host genetics and the outcome of hepatitis B viral infection. Transpl Immunol. 2005;14(3-4):183-6.

11. He Y-L, et al. Host susceptibility to persistent hepatitis B virus infection. WJG. 2006;12(30):4788.
12. Jiang D-K, et al. Genetic variations in STAT4, C2, HLA-DRB1 and HLA-DQ associated with risk of hepatitis B virus-related liver cirrhosis. Sci Reports. 2015:5:16278.

13. Chen VL, et al. Genetic variants that associate with cirrhosis have pleiotropic effects on human traits. Liver Int. 2020;40(2):405-15.

14. Zhang H et al (2010) Genome-wide association study identifies as a new susceptibility locus for hepatocellular carcinoma in chronic hepatitis B virus carriers. Nature Genetics 42(9): 755-758.

15. Li S, et al. GWAS identifies novel susceptibility loci on 6p21. 32 and 21 q21.3 for hepatocellular carcinoma in chronic hepatitis B virus carriers. PLoS Genetics. 2012:8:7.

16. Jiang D-K, et al. Genetic variants in STAT4 and HLA-DQ genes confer risk of hepatitis B virus-related hepatocellular carcinoma. Nat Genet. 2013:45(1):72.

17. Höhler T, et al. Differential genetic determination of immune responsiveness to hepatitis B surface antigen and to hepatitis A virus: a vaccination study in twins. The Lancet. 2002;360(9338):991-5.

18. Ji X, et al. Impacts of human leukocyte antigen DQ genetic polymorphisms and their interactions with hepatitis B virus mutations on the risks of viral persistence, liver cirrhosis, and hepatocellular carcinoma. Infection, Genetics and Evolution. 2014;28:201-9.

19. Zeng Z, et al. A population-based study to investigate host genetic factors associated with hepatitis B infection and pathogenesis in the Chinese population. BMC Infect Dis. 2008;8(1):1.

20. Yang J, et al. GCTA: a tool for genome-wide complex trait analysis. The American Journal of Human Genetics. 2011;88(1):76-82.

21. Purcell S, et al. PLINK: a tool set for whole-genome association and population-based linkage analyses. The American journal of human genetics. 2007:81(3):559-75.

22. Li M-X, et al. Evaluating the effective numbers of independent tests and significant $\mathrm{p}$-value thresholds in commercial genotyping arrays and public imputation reference datasets. Hum Genet. 2012;131(5):747-56.

23. Consortium, G.P. A global reference for human genetic variation. Nature. 2015;526(7571):68-74.

24. De Maio N, Schrempf D, Kosiol C. PoMo: an allele frequency-based approach for species tree estimation. Syst Biol. 2015;64(6):1018-31.

25. Weir BS, Cockerham CC. Estimating F-statistics for the analysis of population structure. Evolution. 1984:1358-1370.

26. Holsinger KE, Weir BS. Genetics in geographically structured populations: defining, estimating and interpreting F ST. Nat Rev Genet. 2009;10(9):639-50.

27. Sabeti $P C$, et al.. Positive natural selection in the human lineage. Science, 2006;312(5780):1614-1620.

28. Voight BF, et al. A map of recent positive selection in the human genome. PLoS Biol. 2006:4(3):e72.

29. Kijas JW, et al. Genome-wide analysis of the world's sheep breeds reveals high levels of historic mixture and strong recent selection. PLoS Biol. 2012;10(2):e1001258.

30. Wang K, et al. Detection of selection signatures in Chinese Landrace and Yorkshire pigs based on genotyping-by-sequencing data. Frontiers in genetics. 2018;9:119.

31. Zhang Z, et al. Whole-genome resequencing reveals signatures of selection and timing of duck domestication. GigaScience, 2018; 7(4):giy027.

32. Danecek $P$, et al. The variant call format and VCFtools. Bioinformatics. 2011:27(15):2156-8.

33. Frankham R, Briscoe DA, Ballou JD. Introduction to conservation genetics. Cambridge University Press, Cambridge; 2002

34. Sabeti $P C$, et al. Detecting recent positive selection in the human genome from haplotype structure. Nature. 2002;419(6909):832-7.

35. Gautier M, Klassmann A, Vitalis R. rehh 2.0: a reimplementation of the $R$ package rehh to detect positive selection from haplotype structure. Mol Ecol Resourc. 2017;17(1):78-90.

36. Speidel $L$, et al. A method for genome-wide genealogy estimation for thousands of samples. Nat Genet. 2019:51(9):1321-9.

37. Chambers JC, et al. Genome-wide association study identifies loci influencing concentrations of liver enzymes in plasma. Nat Genet. 2011:43(11):1131-8.

38. Masuda M, et al. Interaction of genetic markers associated with serum alkaline phosphatase levels in the Japanese population. Human genome variation. 2015;2(1):1-6 
39. Satishchandran A, et al. MicroRNA 122, regulated by GRLH2, protects livers of mice and patients from ethanol-induced liver disease. Gastroenterology. 2018;154(1):238-252.e7.

40. An P, Zeng Z, Winkler CA. The Loss-of-Function S267F variant in HBV receptor NTCP reduces human risk for HBV infection and disease progression. J Infect Dis. 2018;218(9):1404-10.

41. An P, et al. A common HLA-DPA1 variant is a major determinant of hepatitis B virus clearance in Han Chinese. J Infect Dis. 2011;203(7):943-7.

42. Kim SJ, et al. Hepatitis B virus disrupts mitochondrial dynamics: induces fission and mitophagy to attenuate apoptosis. PLoS Pathogens. 2013;9:12.

43. Cesnekova J, et al. The mammalian homologue of yeast Afg1 ATPase (lactation elevated 1) mediates degradation of nuclear-encoded complex IV subunits. Biochem J. 2016;473(6):797-804.

44. Frezza $\mathrm{C}$, et al. OPA1 controls apoptotic cristae remodeling independently from mitochondrial fusion. Cell. 2006;126(1):177-89.

45. Anand R, et al. The $\mathrm{i}-\mathrm{AAA}$ protease $\mathrm{YME} 1 \mathrm{~L}$ and OMA1 cleave OPA1 to balance mitochondrial fusion and fission. J Cell Biol. 2014;204(6):919-29.

46. Kumar PA, SuBramanian K. The role of ischemia modified albumin as a biomarker in patients with chronic liver disease. JCDR. 2016. 10(3): BC09.

47. Yuen $M-F$, et al. Prognostic determinants for chronic hepatitis B in Asians: therapeutic implications. Gut. 2005;54(11):1610-4.

48. Walayat $\mathrm{S}$, et al. Role of albumin in cirrhosis: from a hospitalist's perspective. J Commun Hosp Internal Med Perspect. 2017;7(1):8-14.

49. Castell JV, et al. Interleukin-6 is the major regulator of acute phase protein synthesis in adult human hepatocytes. FEBS Lett. 1989;242(2):237-9.
50. Carvalho JR, Machado MV. New insights about albumin and liver disease. Ann Hepatol. 2018;17(4):547-60.

51. Cichoż-Lach H, Michalak A. Oxidative stress as a crucial factor in liver diseases. WJG. 2014;20(25):8082

52. Teshale EH, et al. Genotypic distribution of hepatitis B virus (HBV) among acute cases of HBV infection, selected United States counties, 1999-2005. Clin Infect Dis. 2011;53(8):751-6.

53. Kim H, et al. Racial/ethnic disparities in the prevalence and awareness of hepatitis B virus infection and immunity in the United States. J Viral Hepatitis. 2017;24(11):1052-66.

54. Tan AT, et al. Host ethnicity and virus genotype shape the hepatitis B virus-specific T-cell repertoire. J Virol. 2008;82(22):10986-97.

55. Guo X, et al. Strong influence of HLA-DP gene variants on development of persistent chronic HBV carriers in the Han Chinese population. Hepatology (Baltimore, Md.), 2011. 53(2):422.

56. Nishida N, et al. Genome-wide association study confirming association of HLA-DP with protection against chronic hepatitis B and viral clearance in Japanese and Korean. PLoS ONE. 2012;7(6):e39175.

57. Wong DKH, et al. Role of HLA-DP polymorphisms on chronicity and disease activity of hepatitis B infection in Southern Chinese. PLoS ONE. 2013;8:6.

\section{Publisher's Note}

Springer Nature remains neutral with regard to jurisdictional claims in published maps and institutional affiliations.
Ready to submit your research? Choose BMC and benefit from:

- fast, convenient online submission

- thorough peer review by experienced researchers in your field

- rapid publication on acceptance

- support for research data, including large and complex data types

- gold Open Access which fosters wider collaboration and increased citations

- maximum visibility for your research: over $100 \mathrm{M}$ website views per year

At BMC, research is always in progress.

Learn more biomedcentral.com/submissions 